\title{
Generalized Thrackle Drawings of Non-bipartite Graphs
}

\author{
Grant Cairns • Yury Nikolayevsky
}

Received: 20 June 2007 / Revised: 22 April 2008 / Accepted: 25 May 2008 /

Published online: 20 June 2008

(C) Springer Science+Business Media, LLC 2008

\begin{abstract}
A graph drawing is called a generalized thrackle if every pair of edges meets an odd number of times. In a previous paper, we showed that a bipartite graph $G$ can be drawn as a generalized thrackle on an oriented closed surface $M$ if and only if $G$ can be embedded in $M$. In this paper, we use Lins' notion of a parity embedding and show that a non-bipartite graph can be drawn as a generalized thrackle on an oriented closed surface $M$ if and only if there is a parity embedding of $G$ in a closed non-orientable surface of Euler characteristic $\chi(M)-1$. As a corollary, we prove a sharp upper bound for the number of edges of a simple generalized thrackle.
\end{abstract}

Keywords Graph drawing · Thrackle

\section{Introduction}

Let $G$ be a finite graph with $n$ vertices and $m$ edges. A drawing of $G$ on a surface $M$ is called a thrackle if every pair of edges of the drawing meets precisely once, either at a vertex or at a proper crossing. Conway's Thrackle Conjecture is that for every thrackle in the plane, $m \leq n$ [19]. The conjecture has apparently been verified for $n \leq 11$; an upper bound $m \leq \frac{3}{2}(n-1)$ was proved in [5, 15]. We conjectured in [5] that $m \leq n+2 g$ for thrackles on a closed oriented surface of genus $g$. To date, this conjecture has only been verified for graphs with $\leq 5$ vertices; see [6].

A natural generalization of the notion of a thrackle is obtained by relaxing the condition that each pair of edges meets precisely once and assuming instead only

G. Cairns $(\bowtie) \cdot$ Y. Nikolayevsky

Department of Mathematics, La Trobe University, Melbourne, Australia 3086

e-mail: G.Cairns@1atrobe.edu.au

Y. Nikolayevsky

e-mail: Y.Nikolayevsky@latrobe.edu.au 
that each pair of edges meets an odd number of times. The resulting notion of a generalized thrackle was introduced by Woodall in 1972 [20]. The following result of [5], which was established in the planar case in [15], effectively classifies generalized thrackles of bipartite graphs, by reducing the problem to a classical embedding problem:

Theorem 1 [5] A finite bipartite graph $G$ can be drawn on an orientable surface $M$ as a generalized thrackle if and only if $G$ can be embedded in $M$.

The aim of this paper is to present an analogous theorem for non-bipartite graphs. This may be regarded as the sequel to [5] and in some sense, a completion of the classification of generalized thrackles.

In order to present the main result, recall that a closed curve $\gamma$ on a surface $M$ is two-sided (resp. one-sided) if the local orientation of the surface is preserved (resp. reversed) as we make one complete circuit of $\gamma$. Recall (see $[14,22])$ :

Definition 1 A parity embedding is a graph embedding $G \rightarrow M$ which maps circuits of even length to two-sided curves, and circuits of odd length to one-sided curves.

Clearly, if a graph $G$ is not bipartite, the image of a parity embedding of $G$ lies on a non-orientable surface. We have:

Theorem 2 A finite connected non-bipartite graph $G$ can be drawn as a generalized thrackle on an oriented closed surface $M$ if and only if $G$ admits a parity embedding in a non-orientable closed surface $N$ with Euler characteristic $\chi(N)=\chi(M)-1$.

This gives the following result which is the generalized thrackle version of Conway's thrackle conjecture:

Corollary 1 For a generalized thrackle drawing of a finite simple graph $G$ with $n$ vertices and $m$ edges on a closed orientable surface $M$ of genus $g$, we have $m \leq$ $2 n-2+4 g$.

This bound was conjectured in [5], where it was established for bipartite graphs; it is sharp, provided that $n$ is reasonably large; see the examples at the end of Sect. 5.

The paper is organized as follows. In Sect. 2, we review some basic terminology and background results. The forward direction of Theorem 2 is proved in Sect. 3, and the converse direction is proved in Sect. 4. Corollary 1 is treated in Sect. 5, and the special case of planar generalized thrackles is treated in Sect. 7. Theorem 2 leaves open the question as to whether the existence of a generalized thrackle has a formulation in terms of embeddings, rather than parity embeddings. We give two results in this direction. Firstly, in Sect. 6, we give a number $k$, which depends only on the graph $G$, for which the following holds (see Theorem 3): if there is a generalized thrackle drawing of $G$ on a closed orientable surface $M$, then there is an embedding of $G$ in a closed orientable surface of Euler characteristic $\chi(M)-k$, and moreover, if there is an embedding of $G$ in a closed orientable surface $M$, then there is a generalized thrackle drawing of $G$ on a closed orientable surface of Euler characteristic 
$\chi(M)-k$. The number $k$ is even and is the rank of a certain 2-form that is trivial when $G$ is bipartite. Secondly, in Sect. 8, we observe that every non-bipartite graph $G$ has a natural bipartite double-cover $\hat{G}$ and prove a result which can be roughly described as follows: $G$ can be drawn as a generalized thrackle on a closed orientable surface $M$ if and only if there is an embedding of $\hat{G}$, with a certain property, in a closed orientable surface of Euler characteristic $2 \chi(M)-2$; see Theorem 5 for details.

Since submitting this paper we have become aware of a preprint by Perlstein and Pinchasi [17]. This interesting paper has several new and original ideas on generalized thrackles. In particular, our Corollary 3 is also proved in [17], by different means.

\section{Terminology and Background Results}

We consider a finite connected (not necessarily simple) graph $G$. By a drawing we mean a smooth immersion of the underlying topological space of $G$ into a surface such that at every vertex $v$, the incident edges have pairwise distinct tangents at $v$. All the drawings of $G$ will be assumed to be "good" in the sense of [15]; that is, the edges are represented by smooth simple curves which are disjoint from the vertex set, every pair of edges in the drawing has only a finite number of intersections, and these are all transversal crossings. It is convenient to define the edges as being open but to define the number of points at which two edges meet as the number of their common crossing points plus the number of any common adjacent vertices (with the convention that the latter number is taken to be zero if at least one of the edges is a loop).

Let $\mathbf{V}$ and $\mathbf{E}$ denote the spaces of formal linear combinations of the vertices (resp. edges) of $G$ with coefficients from $\mathbb{Z}_{2}$; throughout the paper, all the linear spaces, homologies, operators and forms are considered over the field $\mathbb{Z}_{2}$. The boundary operator $\partial: \mathbf{E} \rightarrow \mathbf{V}$ is the $\mathbb{Z}_{2}$-linear operator assigning to each edge the sum of its endpoints. Elements of the subspace $H_{1}(G)=\operatorname{ker} \partial \subset \mathbf{E}$ are called (homological) cycles. This is the usual meaning of cycle in algebraic topology, but cycle has several other distinct meanings in graph theory. In particular, the word cycle is often used for the edge set of an undirected closed path without repeated vertices; to avoid possible confusion, we will call this a circuit, as in [7]. It is easy to see that $H_{1}(G)$ is spanned by circuits. Thus each cycle is a sum of circuits, and topologically, a cycle may be regarded as a union of edge-disjoint circuits. Let $l: \mathbf{E} \rightarrow \mathbb{Z}_{2}$ be the length 1 -form, assigning to a set of edges its cardinality modulo 2 . Using $l$, we can define even and odd paths and circuits in the obvious way. Thus $l$ defines a map from $H_{1}(G)$ to $\mathbb{Z}_{2}$ which we also denote $l$.

We now recall the intersection form on a closed surface $M$. For closed curves $\gamma_{1}, \gamma_{2} \subset M$ in general position, $\Omega_{M}\left(\gamma_{1}, \gamma_{2}\right) \in \mathbb{Z}_{2}$ is the number $(\bmod 2)$ of times $\gamma_{1}$ crosses $\gamma_{2}$. For arbitrary closed curves $\gamma_{1}, \gamma_{2} \subset M$, one first deforms the curves so as to place them in general position; in this way, the number $\Omega_{M}\left(\gamma_{1}, \gamma_{2}\right)$ is well defined and is homotopy-invariant. In fact, as $\Omega_{M}$ takes its values in an abelian group, $\Omega_{M}\left(\gamma_{1}, \gamma_{2}\right)$ actually depends only on $\mathbb{Z}_{2}$-homology classes represented by $\gamma_{1}, \gamma_{2}$, rather than on their homotopy classes. This defines a bilinear form $\Omega_{M}$ : $H_{1}(M) \times H_{1}(M) \rightarrow \mathbb{Z}_{2}$, which is called the $\mathbb{Z}_{2}$-intersection form of $M[9,23]$. The 
intersection form $\Omega_{M}$ is symmetric and if the surface $M$ is orientable, it is alternating; i.e., $\Omega_{M}(\gamma, \gamma)=0$ for all closed curves $\gamma$. Irrespective of whether $M$ is orientable, one has the following standard result, which follows directly from the classification of closed surfaces [18, Theorem 4.4]:

Proposition 1 For a closed surface $M$, the Euler characteristic $\chi(M)=2-\operatorname{rk} \Omega_{M}$.

Remark 1 The definition of the rank $\mathrm{rk} \Omega$ of a form $\Omega$ is not entirely universal; there are two conventions that are adopted in different contexts. For example, consider a symplectic form $\Omega$ on a vector space of dimension $2 k$. On one hand, as a bilinear form, it is common to say that $\Omega$ has rank $k$ since $\Omega$ can be written as the sum of $k$ terms. This is the meaning of rank used in [5]. On the other hand, the rank of a form is often taken to be the rank of the associated matrix, in which case $\Omega$ has rank $2 k$. In this paper we take rank to be the matrix rank. Note that the rank of a non-alternating form may be odd.

Given a drawing $\mathcal{D}: G \rightarrow M$, let $\omega_{\mathcal{D}}$ denote the pull-back of the form $\Omega_{M}$ to $H_{1}(G)$; i.e., $\omega_{\mathcal{D}}\left(c_{1}, c_{2}\right)=\Omega_{M}\left(\mathcal{D}\left(c_{1}\right), \mathcal{D}\left(c_{2}\right)\right)$ for all cycles $c_{1}, c_{2}$ on $G$. Note that this is a common use of the term pull-back in exterior algebra and differentiable geometry (see for example $[3,10]$ ), not to be confused with its other uses, in bundle theory or category theory for example. The following obvious fact could be taken as the definition of a parity embedding:

Lemma 1 An embedding $\mathcal{E}$ of a connected graph $G$ in a closed surface $M$ is a parity embedding if and only if $\omega_{\mathcal{E}}(c, c)=l(c)$ for every circuit $c$ in $G$.

Remark 2 To someone who has not worked with intersection forms before, it may be confusing that for a circuit $c$, the number $\omega_{\mathcal{E}}(c, c)$ may be nonzero. Does not it count the number of intersections of $c$ with itself? And is it not true that circuits do not intersect themselves? The resolution of this apparent contradiction lies in a careful reading of the definition of $\omega_{\mathcal{E}}(c, c)$, which is calculated as follows: take two copies of $c$, then deform them slightly so that they are in general position and hence only meet each other as transverse crossings. Then add the number of these crossing modulo 2 . One sees that $\omega_{\mathcal{E}}(c, c)=1$ precisely when $c$ is orientation reversing.

On an oriented surface $M$, there is another useful alternating 2-form, which is defined as follows. For every drawing $\mathcal{D}: G \rightarrow M$, there is an embedding $\mathcal{E}: G \rightarrow S$ into an oriented surface $S$, which has the same rotation systems as $\mathcal{D}$; the surface $S$ is obtained by attaching a handle to $S$ at each crossing of the drawing. (For information on rotation systems, see [12] or [16].) The 2-form $\omega_{\mathcal{E}}$ depends only on $\mathcal{D}$; we denote it by $\sigma_{\mathcal{D}}$. An explicit description of $\sigma_{\mathcal{D}}$ was given in [5]. We will require the following result which was proved in [5, Lemma 1] (note that this formula still holds when $G$ is a non-simple graph):

Proposition 2 Let $\mathcal{T}$ be a generalized thrackle drawing of a finite graph $G$ on an oriented surface $M$. Then for every pair of cycles $c_{1}, c_{2}$ in $G$, one has:

$$
\omega_{\mathcal{T}}\left(c_{1}, c_{2}\right)=\sigma_{\mathcal{T}}\left(c_{1}, c_{2}\right)+l\left(c_{1}\right) l\left(c_{2}\right)+l\left(c_{1} \cap c_{2}\right) .
$$




\section{Proof of the Forward Direction of Theorem 2}

Suppose that $\mathcal{T}: G \rightarrow M$ is a generalized thrackle drawing of a connected nonbipartite graph $G$ on an oriented closed surface $M$. For every crossing point of $\mathcal{T}(G)$, attach a handle to the surface $M$ in a small neighbourhood of the crossing and reroute the edges to avoid the crossing (as in [5, Fig. 6]). This gives an embedding $\mathcal{T}^{\prime}$ of the graph $G$ in a closed oriented surface $S$ (in general, having much bigger genus than $M$ ). Take a small closed neighbourhood $U \subset S$ of the set $\mathcal{T}^{\prime}(G)$. Then $U$ is an oriented compact surface (with boundary) which consists of small discs around the vertices of $G$ joined by narrow bands along the edges. Take each of these bands in turn, cut across it and glue it back together with a half turn. In this way we obtain a embedding of the graph $G$ in a compact surface $W$ with boundary. Finally, attach discs to all the boundary components of $W$. This results in an embedding $\mathcal{E}$ of the graph $G$ in a closed non-orientable surface $N$. By construction, even circuits of $\mathcal{E}(G)$ represent orientation-preserving loops on $N$, while odd circuits represent orientationreversing ones. So $\mathcal{E}$ is a parity embedding.

For a simple example which illustrates the above construction, consider an embedding of the triangle in the sphere $M=\mathbb{S}^{2}$. This is the simplest possible generalized thrackle of a non-bipartite graph. In performing the above construction, there are no crossings to be removed, and so the compact oriented surface $U$ that we obtain is just a cylinder. Cutting the three bands and regluing, the compact surface $W$ obtained is a Möbius strip. Finally, attaching a disc to the boundary gives $N=\mathbb{R P}^{2}$, the real projective plane.

Returning to the proof of Theorem 2, we will show that $\chi(N) \geq \chi(M)-1$. If necessary, one can then cut out small discs and glue in Möbius strips, to reduce the Euler characteristic of $N$ to $\chi(M)-1$. To begin, consider the pull-backs $\omega_{\mathcal{T}}, \omega_{\mathcal{E}}$ of the respective intersection forms $\Omega_{M}, \Omega_{N}$ to $H_{1}(G)$.

Lemma $2 \omega_{\mathcal{E}}=\omega_{\mathcal{T}}+l \otimes l$.

Proof We must show that for every pair of circuits $c_{1}, c_{2}$ of the graph $G$,

$$
\omega_{\mathcal{E}}\left(c_{1}, c_{2}\right)=\omega_{\mathcal{T}}\left(c_{1}, c_{2}\right)+l\left(c_{1}\right) l\left(c_{2}\right) \text {. }
$$

Formula (2) follows from Lemma 1 when $c_{1}=c_{2}$, since $\mathcal{E}$ is a parity embedding and $\omega_{\mathcal{T}}$ is alternating as $M$ is orientable. So we may assume that $c_{1}$ and $c_{2}$ are distinct. To find the left-hand side of (2) we need to bring the loops $\mathcal{E}\left(c_{1}\right), \mathcal{E}\left(c_{2}\right)$ into general position and to count the number of their crossings mod 2 . This can be achieved by a small perturbation of $\mathcal{E}\left(c_{2}\right)$ in a neighbourhood of the set $\mathcal{E}\left(c_{1} \cap c_{2}\right)$. The intersection $c_{1} \cap c_{2}$ is a disjoint union of paths $p_{1}, \ldots, p_{s}$ and isolated vertices $v_{1}, \ldots, v_{q}$. So we perturb $\mathcal{E}\left(c_{2}\right)$ in a family of disjoint domains, homeomorphic to discs, surrounding the points $\mathcal{E}\left(v_{i}\right)$ and the $\operatorname{arcs} \mathcal{E}\left(p_{j}\right)$ on the surface $N$ for $i=1, \ldots, q, j=1, \ldots, s$.

Now consider the closed oriented surface $U$ described in the construction in the first paragraph of this section. By construction, there is an embedding $\mathcal{P}: G \rightarrow U$ with the same rotation systems as the drawing $\mathcal{T}$ of $G$ in $M$. Consider the pull-back $\omega_{\mathcal{P}}$ of the intersection form $\Omega_{U}$ to $H_{1}(G)$. One has $\omega_{\mathcal{P}}=\sigma_{\mathcal{T}}$. So by Proposition 2, 
we have:

$$
\omega_{\mathcal{T}}\left(c_{1}, c_{2}\right)+l\left(c_{1}\right) l\left(c_{2}\right)=\omega_{\mathcal{P}}\left(c_{1}, c_{2}\right)+l\left(c_{1} \cap c_{2}\right) .
$$

So (2) is equivalent to

$$
\omega_{\mathcal{E}}\left(c_{1}, c_{2}\right)=\omega_{\mathcal{P}}\left(c_{1}, c_{2}\right)+l\left(c_{1} \cap c_{2}\right) .
$$

At every isolated vertex $v_{i}$ of $c_{1} \cap c_{2}$, the loops $\mathcal{E}\left(c_{1}\right)$ and $\mathcal{E}\left(c_{2}\right)$ on $N$ touch or cross in the same manner as $\mathcal{P}\left(c_{1}\right)$ and $\mathcal{P}\left(c_{2}\right)$ do on $U$. So the contribution of each $v_{i}$ to both sides of (3) is the same.

For each path $p_{j}$, the band on $N$ surrounding $\mathcal{E}\left(p_{j}\right)$ makes $l\left(p_{j}\right)$ half-turns relative to the same band on $U$. Therefore, the number of crossing points of the loop $\mathcal{E}\left(c_{1}\right)$ and the perturbed loop $\mathcal{E}\left(c_{2}\right)$ in the band on $N$ is $l\left(p_{j}\right)$ more than the corresponding number of crossings on $U$. In other words, for the path $p_{j}$, the contribution to the right-hand side of $(3)$ is $l\left(p_{j}\right)$ more than the corresponding contribution to the left-hand side of (3). This establishes (3) and completes the proof of the lemma.

By construction, $\mathrm{rk} \omega_{\mathcal{T}} \leq \mathrm{rk} \Omega_{M}$. So, as $\chi(M)=2-\mathrm{rk} \Omega_{M}$ by Proposition 1 , we have $\chi(M) \leq 2-\operatorname{rk} \omega_{\mathcal{T}}$. Since the embedding $\mathcal{E}$ is cellular, $\chi(N)=2-$ rk $\omega_{\mathcal{E}}$. Thus, in order to establish $\chi(N) \geq \chi(M)-1$, the following elementary exercise in linear algebra will suffice.

Lemma 3 Given an alternating bilinear form $\omega_{\mathcal{T}}$ and a 1-form $l$, in a vector space $L$ over $\mathbb{Z}_{2}$, set $\omega_{\mathcal{E}}=\omega_{\mathcal{T}}+l \otimes l$. Then either $\operatorname{rk} \omega_{\mathcal{E}}=\operatorname{rk} \omega_{\mathcal{T}}$ or $\operatorname{rk} \omega_{\mathcal{E}}=\operatorname{rk} \omega_{\mathcal{T}}+1$.

Proof If $l=0$, there is nothing to prove. Otherwise, let $p=\operatorname{dim} L$ and choose a basis $c_{1}, \ldots, c_{p}$ of $L$ such that $c_{1}, \ldots, c_{p-1}$ span the kernel of $l$. As $\omega_{\mathcal{T}}$ is alternating, the restriction $\omega_{\mathcal{T}}^{\prime}$ of $\omega_{\mathcal{T}}$ to $\operatorname{ker} l$ is also alternating. In particular, $\operatorname{rk}\left(\omega_{\mathcal{T}}^{\prime}\right)$ is even. Set $\operatorname{rk} \omega_{\mathcal{T}}^{\prime}=2 k$ and consider the standard $2 k \times 2 k \mathbb{Z}_{2}$-symplectic matrix $J_{k}$ :

$$
J_{k}=\left(\begin{array}{cccc}
J & 0 & \ldots & 0 \\
0 & J & \ldots & 0 \\
\vdots & \vdots & \ddots & \vdots \\
0 & 0 & \ldots & J
\end{array}\right), \quad \text { where } J=\left(\begin{array}{cc}
0 & 1 \\
1 & 0
\end{array}\right)
$$

which consists of $k$ copies of $J$ down the main diagonal and zeros elsewhere. By [13, Theorem 2.10], since $\omega_{\mathcal{T}}^{\prime}$ is alternating, we can specify the vectors $c_{1}, \ldots, c_{p-1}$ in such a way that the matrix representation of $\omega_{\mathcal{T}}^{\prime}$ is:

$$
\left(\begin{array}{cc}
J_{k} & 0 \\
0 & 0
\end{array}\right)
$$

Thus the matrix representations of $\omega_{\mathcal{T}}$ and $\omega_{\mathcal{E}}$ are respectively

$$
\left(\begin{array}{ccc}
J_{k} & 0 & \alpha^{t} \\
0 & 0 & \beta^{t} \\
\alpha & \beta & 0
\end{array}\right) \quad \text { and } \quad\left(\begin{array}{ccc}
J_{k} & 0 & \alpha^{t} \\
0 & 0 & \beta^{t} \\
\alpha & \beta & 1
\end{array}\right)
$$


for some $\alpha=\left(\alpha_{1}, \ldots, \alpha_{2 k}\right), \beta=\left(\beta_{1}, \ldots, \beta_{p-2 k-1}\right)$. Changing $c_{p}$ to $c_{p}+\alpha_{1} c_{2}+$ $\alpha_{2} c_{1}+\cdots+\alpha_{2 k-1} c_{2 k}+\alpha_{2 k} c_{2 k-1}$, we obtain matrices of the same form with the $\alpha_{i}$ replaced by zeros. If $\beta_{j}=0$ for all $j$, then $\operatorname{rk} \omega_{\mathcal{E}}=\operatorname{rk} \omega_{\mathcal{T}}+1$. If at least one of the $\beta_{j}$ is nonzero, $\operatorname{rk} \omega_{\mathcal{E}}=\operatorname{rk} \omega_{\mathcal{T}}$. This completes the proof of Lemma 3 and the forward direction of Theorem 2.

\section{Proof of the Converse Direction of Theorem 2}

We will show that the construction of the previous section can be reversed; i.e., for each parity embedding of $G$ in a closed non-orientable surface $N$ of odd Euler characteristic $\chi(N)$, there is a generalized thrackle drawing of $G$ in an orientable closed surface $M$ with $\chi(M) \geq \chi(N)+1$.

Assume that $\mathcal{E}: G \rightarrow N$ is a parity embedding of a graph $G$ in a closed surface $N$. Consider a small closed neighbourhood $W \subset N$ of $\mathcal{E}(G)$. So $W$ is a compact surface with boundary, consisting of discs around the vertices of $G$ and bands around the edges. Cut each of these bands across and then glue back with a half turn. This gives an orientable surface $U$ with boundary and an embedding $\mathcal{P}: G \rightarrow U$. Attach discs to each of the boundary component. The resulting closed surface $S$ is orientable, with the graph $G$ embedded by a map $\mathcal{S}: G \rightarrow S$. In general, we cannot control the Euler characteristic of $S$. However, the forms $\omega_{\mathcal{S}}$ and $\omega_{\mathcal{E}}$ are closely related.

Lemma 4 For every pair of cycles $c_{1}, c_{2}$ in $G$,

$$
\omega_{\mathcal{S}}\left(c_{1}, c_{2}\right)=\omega_{\mathcal{P}}\left(c_{1}, c_{2}\right)=\omega_{\mathcal{E}}\left(c_{1}, c_{2}\right)+l\left(c_{1} \cap c_{2}\right) .
$$

Proof The first equation of (4) follows from the fact that the embedding $\mathcal{S}$ is cellular. The second one trivially holds when $c_{1}=c_{2}$, by the definition of a parity embedding. If $c_{1} \neq c_{2}$, perturb the images $\mathcal{E}\left(c_{1}\right), \mathcal{E}\left(c_{2}\right) \subset N$ in a neighbourhood of $\mathcal{E}\left(c_{1} \cap c_{2}\right)$ to bring them into general position. Then $\omega_{\mathcal{E}}\left(c_{1}, c_{2}\right)$ is the number of crossings of the resulting loops modulo 2 . Under the embedding $\mathcal{P}$, the images of cycles $c_{1}, c_{2}$ acquire additional crossings, one for every edge the cycles have in common. Thus, arguing as in the proof of Lemma 2, one has $\omega_{\mathcal{P}}\left(c_{1}, c_{2}\right)=\omega_{\mathcal{E}}\left(c_{1}, c_{2}\right)+l\left(c_{1} \cap c_{2}\right)$.

In what follows, the strategy is not to attempt to construct the required drawing $\mathcal{T}: G \rightarrow M$ by performing surgery on $S$ but rather to construct $M$ from scratch, so to speak, using only the rotations systems of $\mathcal{S}: G \rightarrow S$. We will use the following proposition, which is a converse to Proposition 2 .

Proposition 3 Let $\mathcal{D}$ be a drawing of a finite graph $G$ on an oriented surface $M$, and suppose furthermore that for every pair of cycles $c_{1}, c_{2}$ in $G$, one has:

$$
\omega_{\mathcal{D}}\left(c_{1}, c_{2}\right)=\sigma_{\mathcal{D}}\left(c_{1}, c_{2}\right)+l\left(c_{1}\right) l\left(c_{2}\right)+l\left(c_{1} \cap c_{2}\right) .
$$

Then there exists a generalized thrackle drawing $\mathcal{T}: G \rightarrow M$ with the same rotation diagram and the same homologies of cycles as in the drawing $\mathcal{D}$. 
We first complete the proof of Theorem 2 assuming Proposition 3. Consider the alternating two-form $\omega$ defined on $H_{1}(G)$ by

$$
\omega\left(c_{1}, c_{2}\right)=\omega_{\mathcal{S}}\left(c_{1}, c_{2}\right)+l\left(c_{1} \cap c_{2}\right)+l\left(c_{1}\right) l\left(c_{2}\right)
$$

for cycles $c_{1}, c_{2}$ in $G$. Set $2 g=\operatorname{rk} \omega$ and let $M$ be a closed oriented surface of genus $g$. Choose an epimorphism $\phi: H_{1}(G) \rightarrow H_{1}(M) \simeq \mathbb{Z}_{2}^{2 g}$ such that $\omega=\phi^{*} \Omega_{M}$; that is, $\omega\left(c_{1}, c_{2}\right)=\Omega_{M}\left(\phi\left(c_{1}\right), \phi\left(c_{2}\right)\right)$ for all cycles $c_{1}, c_{2}$. This is always possible since $\Omega_{M}$ is non-degenerate; see [13, Theorem 2.10]. We now construct a drawing $\mathcal{D}$ of $G$ on $M$ with the following properties:

(1) the rotation diagram of $\mathcal{D}$ is the same as that of the embedding $\mathcal{S}$ of $G$ in $S$,

(2) for every cycle $c, \mathcal{D}(c)$ represents $\phi(c)$ in $H_{1}(M)$.

To construct such a drawing, we start by embedding a spanning tree of $G$ respecting the rotation diagram and then add the remaining edges respecting both the rotation diagram and homologies of cycles.

Applying Proposition 3, since $\omega_{\mathcal{S}}=\sigma_{\mathcal{D}}$, we can redraw $\mathcal{D}$ to get a generalized thrackle drawing $\mathcal{T}: G \rightarrow M$. By (4) and (6), $\omega\left(c_{1}, c_{2}\right)=\omega_{\mathcal{E}}\left(c_{1}, c_{2}\right)+l\left(c_{1}\right) l\left(c_{2}\right)$ for cycles $c_{1}, c_{2}$ in $G$; in other words, $\omega_{\mathcal{E}}=\omega+l \otimes l$. Hence by Lemma 3 , rk $\omega \leq \operatorname{rk} \omega_{\mathcal{E}}$. Thus $\chi(M)=2-\mathrm{rk} \omega \geq 2-\mathrm{rk} \omega_{e}=\chi(N)$. So, as $\chi(M)$ is even and $\chi(N)$ is odd, $\chi(M) \geq \chi(N)+1$, as required.

It remains to give the:

Proof of Proposition 3 Consider an arbitrary edge $e$ and vertex $v$ of $G$. Define an $(e, v)$-operation on the drawing $\mathcal{D}$ as shown in Fig. 1. Since an $(e, v)$-operation changes neither rotation diagrams nor homologies, it leaves (5) unchanged. We propose to turn $\mathcal{D}$ into a generalized thrackle in a finite number of $(e, v)$-operations.

Consider the boundary map $\partial: \mathbf{E} \rightarrow \mathbf{V}$ between the edge and vertex spaces of $G$. We introduce an alternating $\mathbb{Z}_{2}$-bilinear 2-form $\eta$ on $\mathbf{E}$ as follows: $\eta(e, e)=0$ for all edges $e$, and for distinct edges $e_{1}, e_{2}$,

$$
\eta\left(e_{1}, e_{2}\right)=1+\#\left(\mathcal{D}\left(e_{i}\right) \cap \mathcal{D}\left(e_{j}\right)\right)+\#\left(\partial\left(e_{1}\right) \cap \partial\left(e_{2}\right)\right) \quad(\bmod 2) .
$$

As usual, edges are by definition open edges, so the intersection $\mathcal{D}\left(e_{1}\right) \cap \mathcal{D}\left(e_{2}\right)$ does not include endpoints; the number of common endpoints is counted in the third term $\#\left(\partial\left(e_{1}\right) \cap \partial\left(e_{2}\right)\right)$ (which we take to be zero if at least one of the edges $e_{1}, e_{2}$ is a loop). Notice that the drawing $\mathcal{D}$ is a generalized thrackle if and only if $\eta \equiv 0$.

Lemma 5 For an arbitrary graph drawing $\mathcal{D}$ and for all cycles $c_{1}, c_{2}$ of $G$,

$$
\eta\left(c_{1}, c_{2}\right)=l\left(c_{1}\right) l\left(c_{2}\right)+l\left(c_{1} \cap c_{2}\right)+\omega_{\mathcal{D}}\left(c_{1}, c_{2}\right)+\sigma_{\mathcal{D}}\left(c_{1}, c_{2}\right) \quad(\bmod 2) .
$$

Proof If $A_{i}$ denotes the set of indices $j$ for which edges $e_{j}$ form part of $c_{i}$, then modulo 2,

$$
\eta\left(c_{1}, c_{2}\right)=\sum_{\substack{i \in A_{1}, j \in A_{2} \\ i \neq j}} 1+\#\left(\mathcal{D}\left(e_{i}\right) \cap \mathcal{D}\left(e_{j}\right)\right)+\#\left(\partial\left(e_{i}\right) \cap \partial\left(e_{j}\right)\right)
$$



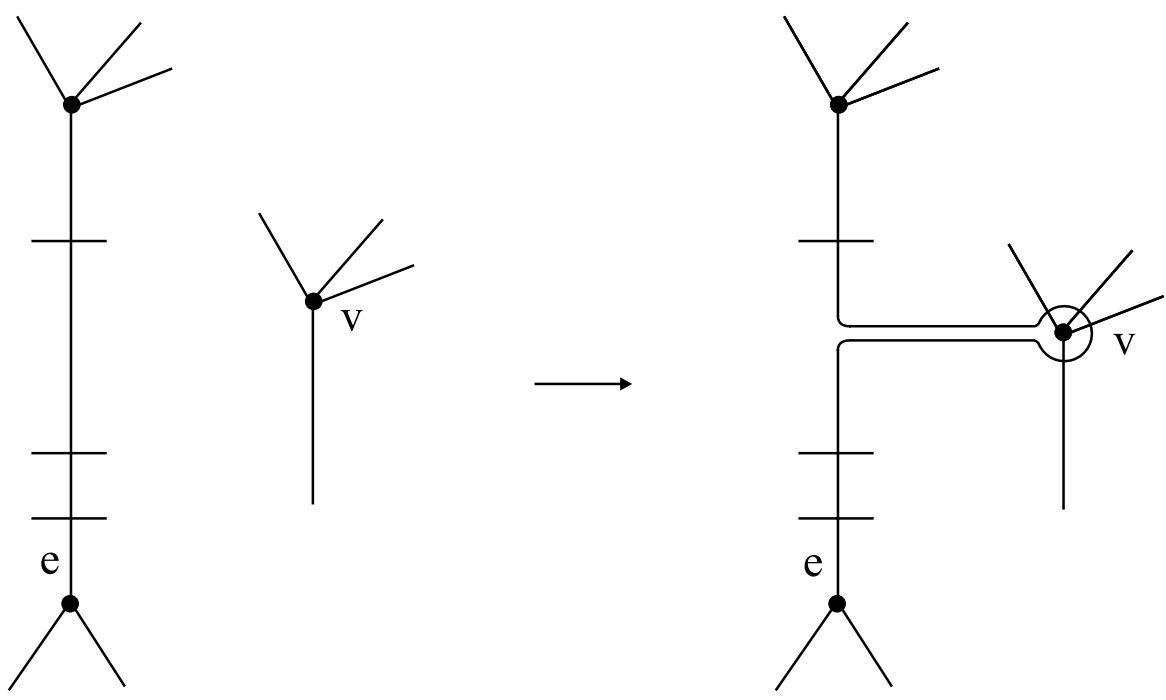

Fig. 1 An $(e, v)$-operation

$$
\begin{aligned}
& =\#\left(\partial\left(c_{1}\right) \cap \partial\left(c_{2}\right)\right)+\sum_{\substack{i \in A_{1}, j \in A_{2} \\
i \neq j}} 1+\#\left(\mathcal{D}\left(e_{i}\right) \cap \mathcal{D}\left(e_{j}\right)\right) \\
& =\sum_{\substack{i \in A_{1}, j \in A_{2} \\
i \neq j}} 1+\#\left(\mathcal{D}\left(e_{i}\right) \cap \mathcal{D}\left(e_{j}\right)\right) \\
& =l\left(c_{1}\right) l\left(c_{2}\right)+l\left(c_{1} \cap c_{2}\right)+\sum_{\substack{i \in A_{1}, j \in A_{2} \\
i \neq j}} \#\left(\mathcal{D}\left(e_{i}\right) \cap \mathcal{D}\left(e_{j}\right)\right) \\
& =l\left(c_{1}\right) l\left(c_{2}\right)+l\left(c_{1} \cap c_{2}\right)+\omega_{\mathcal{D}}\left(c_{1}, c_{2}\right)+\sigma_{\mathcal{D}}\left(c_{1}, c_{2}\right),
\end{aligned}
$$

where the last line uses the fact that, by the definition of $\omega_{\mathcal{D}}$ and $\sigma_{\mathcal{D}}, \omega_{\mathcal{D}}\left(c_{1}, c_{2}\right)+$ $\sigma_{\mathcal{D}}\left(c_{1}, c_{2}\right)$ is the number (mod 2) of crossings of $\mathcal{D}\left(c_{1}\right)$ with $\mathcal{D}\left(c_{2}\right)$ that occur in the interior of the edges.

Notice that by the above lemma, the hypothesis of Proposition 3 reads as follows: $\eta\left(c_{1}, c_{2}\right)=0$ for all cycles $c_{1}, c_{2}$. The subspace of cycles in $\mathbf{E}$ is just the kernel of the boundary map $\partial: \mathbf{E} \rightarrow \mathbf{V}$. So the condition that $\eta\left(c_{1}, c_{2}\right)=0$ for all cycles $c_{1}, c_{2}$ can be rephrased as: $\operatorname{ker} \partial$ is totally isotropic for the alternating form $\eta$.

Label the vertices $v_{1}, \ldots, v_{n}$ and edges $e_{1}, \ldots, e_{m}$ and take these as bases for $\mathbf{V}$ and $\mathbf{E}$ respectively. Relative to these bases, let $d$ be the matrix representation of $\partial$, and let $D$ be the matrix representation of $\eta$. Notice that the $\left(e_{i}, v_{j}\right)$-operation adds the $j$ th row of $d$ to both the $i$ th row and the $i$ th column of $D$. Take a $\mathbb{Z}_{2}$-matrix $P$ with $m$ rows and $n$ columns and perform the $\left(e_{i}, v_{j}\right)$-operations on each pair $\left(e_{i}, v_{j}\right)$ such that $P_{i j}=1$. Then the matrix $D$ for the resulting drawing has the form $D+P d+(P d)^{t}$. So Proposition 3 is equivalent to the following fact of matrix algebra: 
Lemma 6 If the kernel of an $n \times m$-matrix $d$ is totally isotropic for an alternating $m \times m$-matrix $D$, then there exists an $m \times n$-matrix $P$ such that

$$
D+P d+(P d)^{t}=0 .
$$

Proof By change of basis in $\mathbb{Z}_{2}^{m}$ and $\mathbb{Z}_{2}^{n}$, we may assume that the matrix $d$ has the form $d=\left(\begin{array}{cc}I_{r} & 0 \\ 0 & 0\end{array}\right)$, where $r=\operatorname{rk} d$, and $I_{r}$ is the $r \times r$ identity matrix. Then $D=$ $\left(\begin{array}{cc}D_{1} & D_{2} \\ D_{2}^{t} & 0\end{array}\right)$, where the $r \times r$ matrix $D_{1}$ is alternating, and $D_{2}$ is an $r \times(m-r)$-matrix. Thus (7) holds with $P=\left(\begin{array}{cc}P_{1} & 0 \\ D_{2}^{t} & 0\end{array}\right)$, where $P_{1}$ is the strictly upper-triangular $r \times r$ matrix having the same elements above the diagonal as $D_{1}$.

This completes the proof of the lemma, Proposition 3, and Theorem 2.

\section{Proof of Corollary 1 and the Sharpness of the Inequality}

Suppose that $\mathcal{T}: G \rightarrow M$ is a generalized thrackle drawing, where $M$ is a closed orientable surface of genus $g$. Then by Theorem 2 , there is a parity embedding $\mathcal{E}: G \rightarrow N$, where $\chi(N)=\chi(M)-1=1-2 g$. Further, by reducing the genus if necessary, we can assume that $\mathcal{E}$ is cellular. For each face $F$ of $\mathcal{E}$, the boundary $\partial F$ of $F$ is a 2 -sided curve and so, as $\mathcal{E}$ is a parity embedding, the boundary $\partial F$ must consist of an even number of edges; i.e., $F$ is a $2 m$-gon for some $m$. (We remark in passing that it is possible that an edge may occur twice in the boundary of a given face, as for example in the case of the parity embedding of the triangle in $\mathbb{R} P^{2}$ described in Sect. 3. In this example, there is a single hexagonal face.) Hence, if $G$ is a simple graph, the faces of $N$ all have at least 4 edges. It follows from Euler's formula that $m \leq 2 n-2+4 g$, where $n$ is the number of vertices of $G$, and $m$ is the number of edges. This establishes Corollary 1 .

Applying Theorem 2, we can now show the sharpness of the bound given in Corollary 1. First take a minimal genus embedding of a complete bipartite graph $K_{4,2 q}$, $q \geq 1$ in a closed orientable surface $M$ of genus $g$. Then $m=8 q, n=2 q+4$ and $g=q-1$ [12]. So $m=2 n+4 g-4$. All the faces of the embedding are quadrilaterals. Let $D$ be one of the faces and $v_{1}, v_{2}, v_{3}, v_{4}$ its vertices. Cut the face $D$ out and attach a Möbius band along the boundary circle of $D$. We can draw two arcs $v_{1} v_{3}$ and $v_{2} v_{4}$ on this band such that the resulting drawing is still an embedding. Moreover, this embedding is a parity embedding. The resulting graph $G$ has $m^{\prime}=m+2$ edges and $n^{\prime}=n$ vertices and is parity embedded in a surface of Euler characteristic $1-2 g$. By Theorem 2, we can redraw $G$ as a generalized thrackle on a closed orientable surface of Euler characteristic $2-2 g$. This results in a generalized thrackle with $m^{\prime}=2 n^{\prime}+4 g-2$.

Note that having a generalized thrackle for which the equality sign in Corollary 1 is attained, we can produce new generalized thrackles on the same surface without violating the equality. This can be done by adding 2-paths as shown in [5, Fig. 10]. 


\section{Embedding Conditions}

For an arbitrary finite graph $G$, let $\Lambda$ denote the 2-form on $H_{1}(G)$ defined by:

$$
\Lambda\left(c_{1}, c_{2}\right)=l\left(c_{1}\right) l\left(c_{2}\right)+l\left(c_{1} \cap c_{2}\right) \quad(\bmod 2)
$$

for all cycles $c_{1}, c_{2}$. It is clear that $\Lambda$ is $\mathbb{Z}_{2}$-bilinear and alternating.

Theorem 3 If there is a generalized thrackle drawing of $G$ in a closed orientable surface $M$, then there is an embedding of $G$ in a closed orientable surface of Euler characteristic $\chi(M)-\operatorname{rk}(\Lambda)$ and moreover, if there is an embedding of $G$ in a closed orientable surface $S$, then there is a generalized thrackle drawing of $G$ in a closed orientable surface of Euler characteristic $\chi(S)-\operatorname{rk}(\Lambda)$.

Proof First suppose that $\mathcal{T}: G \rightarrow M$ is a generalized thrackle drawing, where $M$ is a closed orientable surface. Let $\mathcal{E}: G \rightarrow S$ be an embedding in a closed oriented surface $S$ with the same rotation systems as $\mathcal{T}$. Then $\omega_{\mathcal{E}}=\sigma_{\mathcal{T}}$. So by Proposition 1 , $\omega_{\mathcal{E}}=\omega_{\mathcal{T}}+\Lambda$ and hence $\operatorname{rk}\left(\omega_{\mathcal{E}}\right) \leq \operatorname{rk}\left(\omega_{\mathcal{T}}\right)+\operatorname{rk}(\Lambda)$. Moreover, arguing as in the proof of [5, Lemma 3], we can choose $S$ to have minimal genus so that $\operatorname{rk}\left(\omega_{\mathcal{E}}\right)=\operatorname{rk}\left(\Omega_{S}\right)$. Then by Proposition 1,

$$
\chi(S)=2-\operatorname{rk}\left(\Omega_{S}\right)=2-\operatorname{rk}\left(\omega_{\mathcal{E}}\right) \geq 2-\operatorname{rk}\left(\omega_{\mathcal{T}}\right)-\operatorname{rk}(\Lambda)=\chi(M)-\operatorname{rk}(\Lambda) .
$$

Conversely, suppose that $\mathcal{E}: G \rightarrow S$ is an embedding of $G$ in a closed oriented surface $S$. Arguing as in Sect. 4, there is a generalized thrackle drawing $\mathcal{T}: G \rightarrow M$ with $\sigma_{\mathcal{T}}=\omega_{\mathcal{E}}$, where $M$ a closed oriented surface with $\chi(M)=2-\operatorname{rk}\left(\omega_{\mathcal{E}}\right)$. Then by Proposition $2, \omega_{\mathcal{T}}=\omega_{\mathcal{E}}+\Lambda$. Hence $\operatorname{rk}\left(\omega_{\mathcal{T}}\right) \leq \operatorname{rk}\left(\omega_{\mathcal{E}}\right)+\operatorname{rk}(\Lambda)$, and so

$$
\chi(M)=2-2 \operatorname{rk}\left(\omega_{\mathcal{T}}\right) \geq 2-\operatorname{rk}\left(\omega_{\mathcal{E}}\right)-\operatorname{rk}(\Lambda)=\chi(S)-\operatorname{rk}(\Lambda),
$$

as required.

Notice that if $G$ is bipartite, then $\Lambda \equiv 0$. Thus Theorem 3 is a generalization of Theorem 1.

\section{Planar Generalized Thrackles}

Theorem 2 has the following immediate corollary:

Corollary 2 A finite connected non-bipartite graph $G$ can be drawn as a generalized thrackle in the plane if and only if $G$ admits a parity embedding in $\mathbb{R} \mathbb{P}^{2}$.

There is a Kuratowski-type classification theorem for embeddings of graphs in the projective plane [1], but there is no analogous result for parity embeddings [21]. Of course, the condition that there is a parity embedding a graph $G$ in $\mathbb{R P}^{2}$ is much stronger than the condition that $G$ embeds in $\mathbb{R P}^{2}$. Indeed, it is easy to construct 
graphs which are planar (and hence embed in $\mathbb{R P}^{2}$ ) but which do not possess a parity embedding in $\mathbb{R P}^{2}$; see Sect. 8 .

Consider the following construction: if $G$ is a graph and $c$ an even circuit in $G$, define the equivalence relation $\sim_{c}$ on $G$ which identifies opposite edges and opposite vertices of $c$ and is trivial outside $c$. We have:

Theorem 4 There is a parity embedding of a finite connected non-bipartite graph $G$ in the projective plane $\mathbb{R P}^{2}$ if and only if there exist a planar bipartite graph $G^{\prime}$ and a circuit $c \subset G^{\prime}$ of length 2 (mod 4) bounding a face in a plane embedding of $G^{\prime}$ such that $G=G^{\prime} / \sim_{c}$.

Proof If there is a parity embedding of $G$ in $\mathbb{R P}^{2}$, then by Corollary $1, G$ can be drawn as a generalized thrackle in the plane. Conway doubling on an odd circuit gives a planar generalized thrackle of a graph of the required form $G^{\prime}$ (see [19]). Moreover, $G^{\prime}$ is bipartite [5, Lemma 2]. Hence $G$ embeds in the plane, by [15] (see Theorem 1).

Conversely, suppose that $G^{\prime}$ is a planar bipartite graph and $c^{\prime}$ is a circuit of length $2(\bmod 4)$ bounding a face $D$ in an embedding $\mathcal{F}: G^{\prime} \rightarrow \mathbb{S}^{2}$. Since $c^{\prime}$ is a circuit, the complement $\mathbb{S}^{2} \backslash D$ is a disc. Cut the face $D$ out and identify the opposite points of its boundary circle $c^{\prime}$. We obtain an embedding $\mathcal{E}$ of the graph $G=G^{\prime} / \sim_{c^{\prime}}$ in $\mathbb{R P}^{2}$. It remains to show that $\mathcal{E}$ is a parity embedding. By construction, the diagram

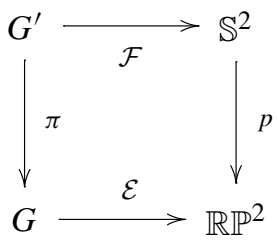

is commutative with $\pi: G^{\prime} \rightarrow G$ the projection operator defined by the equivalence relation $\sim_{c^{\prime}}$ and $p$ the projection from $\mathbb{S}^{2}$ to $\mathbb{R} \mathbb{P}^{2}$. The induced maps in homology give the commutative diagram:

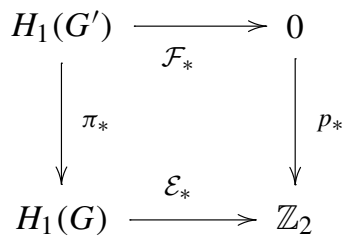

Let $c$ denote the odd circuit in $G$ that results from $c^{\prime}$; that is, $\pi\left(c^{\prime}\right)=c$. As $\pi: c^{\prime} \rightarrow c$ is a double-cover, $\pi_{*}\left(c^{\prime}\right)=0$; in fact, it is easy to see that the kernel of $\pi_{*}$ is the one-dimensional subspace spanned by $c^{\prime}$. Let $n$ (resp. $n^{\prime}$ ) and $m$ (resp. $m^{\prime}$ ) denote respectively the number of vertices and edges of $G$ (resp. $G^{\prime}$ ). Recall that for an arbitrary connected graph $K$ with $v$ vertices and $e$ edges, one has $\operatorname{rk} H_{1}(K)=e-$ $v+1$, the cyclomatic number of $K$; see [11]. Since $n-m=n^{\prime}-m^{\prime}$, one has 
Fig. 2 Conway doubling for parity embeddings

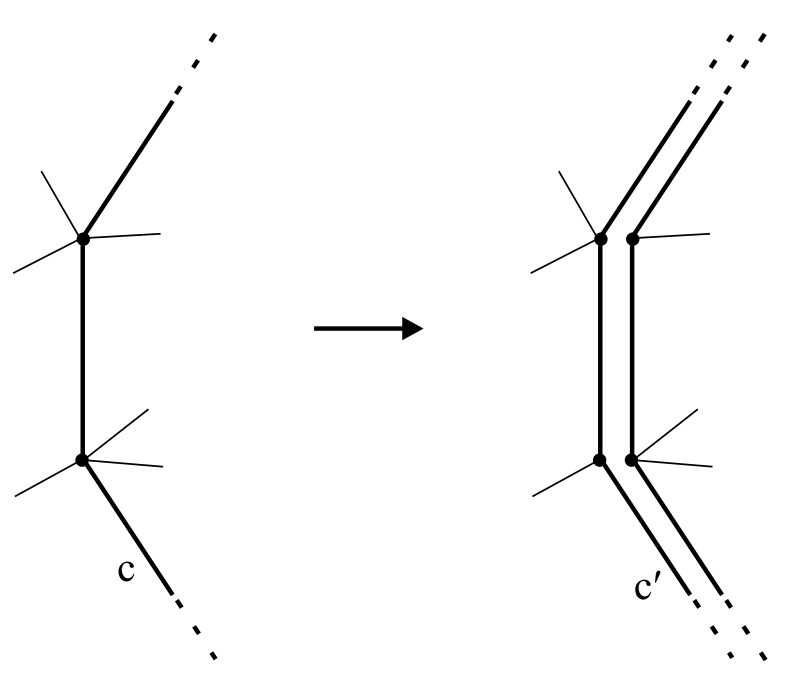

rk $H_{1}\left(G^{\prime}\right)=$ rk $H_{1}(G)$. Since $\pi$ maps edges onto edges, the homomorphism $\pi_{*}$ respects parity; i.e., on $H_{1}\left(G^{\prime}\right), l \circ \pi_{*}=l$, which is identically zero, as $G^{\prime}$ is bipartite. Thus, the cycles in $\operatorname{Im}\left(\pi_{*}\right)$ are even. Hence $c \notin \operatorname{Im}\left(\pi_{*}\right)$, and it follows that $H_{1}(G)$ is spanned by $\operatorname{Im}\left(\pi_{*}\right)$ and $c$. Consequently, the even cycles in $G$ all lie in $\operatorname{Im}\left(\pi_{*}\right)$ and are therefore zero in $H_{1}\left(\mathbb{R}^{2}\right)$, by the above commutative diagram. The odd circuit $c$ represents an orientation-reversing loop, and is therefore nontrivial in homology. Thus, as every odd circuit has the form $c+\gamma$, where $\gamma \in \operatorname{Im}\left(\pi_{*}\right)$, all odd circuits are nontrivial in homology; i.e., they are 1 -sided. So $\mathcal{E}$ is a parity embedding.

Note that in the first part of the above proof, we could have argued directly without recourse to the results of [5]. Indeed, given an parity embedding $\mathcal{E}: G \rightarrow \mathbb{R}^{2}$, one can perform a construction which may be regarded as a form of "Conway doubling" for parity embeddings: choose an odd circuit $c$ in $G$. Double each of the vertices and edges of $c$, as shown in Fig. 2. In this way, since $c$ is single sided, $c$ is replaced by a circuit $c^{\prime}$ of twice the length as $c$; let $G^{\prime}$ denote the resulting graph. The circuit $c^{\prime}$ bounds a Möbius strip; cutting this out and replacing it by a disc we obtain an embedding of $G^{\prime}$ in $\mathbb{S}^{2}$, and clearly $G=G^{\prime} / \sim_{c}$. The only work that remains is to show that $G^{\prime}$ is bipartite; this requires an argument similar to that in [5, Lemma 2].

\section{Parity Embeddings}

We conclude the paper with some comments concerning parity embeddings. First recall that if $G$ is a non-bipartite connected graph, then there is a natural bipartite graph $\hat{G}$ and a fixed point free action of the two element group $\mathbb{Z}_{2}$ on $\hat{G}$ with $G=\hat{G} / \mathbb{Z}_{2}$ (see [2]). The graph $\hat{G}$ is called the bipartite double-cover of $G$; it is analogous to the orientation double-cover of a non-orientable surface and is constructed as follows: consider the length function $l: \pi_{1}(G) \rightarrow \mathbb{Z}_{2}$ on the fundamental group $\pi_{1}(G)$. Clearly $l$ is a group homomorphism, and it is nontrivial as $G$ is non-bipartite. Let 
Fig. 3 The complete graph $K_{4}$ and its bipartite double-cover
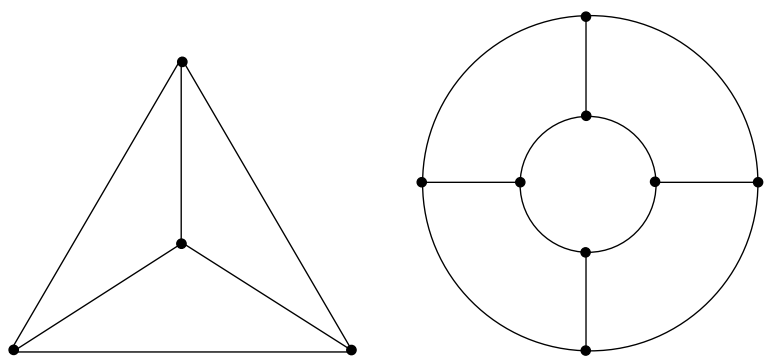

$p: \hat{G} \rightarrow G$ denote the Galois covering of the topological space $G$ corresponding to the homomorphism $l$. (Recall that a covering is said to be Galois or regular if it is defined by a free action of a group [8, Chap. 3.I].) Then $\hat{G}$ inherits a structure of a graph from $G$, which is clearly bipartite by construction, and the monodromy action of $\mathbb{Z}_{2}$ on $\hat{G}$ is the required $\mathbb{Z}_{2}$ action. From a practical point of view, $\hat{G}$ can be constructed as follows: remove from $G$ the minimum possible number of edges so as to make the resulting graph bipartite. Take two copies $G_{1}, G_{2}$ of this bipartite graph and two copies of the removed edges and reattach the edges to their original vertices but in such a way that each attached edge joins $G_{1}$ with $G_{2}$. The graph thus constructed is $\hat{G}$.

In the planar case, Theorem 2 can be rephrased as follows:

Corollary 3 A finite connected non-bipartite graph $G$ can be drawn as a generalized thrackle on the plane if and only if its bipartite double-cover $\hat{G}$ can be embedded in the complex plane in such a manner that the monodromy map is the antipodal map $z \mapsto-1 / z^{*}$.

For example, consider the graph $G=K_{4}$, shown on the left of Fig. 3. Its bipartite double-cover $\hat{G}$ is the cube; the planar embedding of $\hat{G}$, shown on the right of Fig. 3 , is to be regarded as being centred at the origin in the complex plane $\mathbb{C}$, and the monodromy map is the antipodal map $z \mapsto-1 / z^{*}$. So by Corollary $3, G$ can be drawn as a generalized thrackle in the plane; see [5, Fig. 10].

Notice that in general, if there is a parity embedding of a graph $G$ in a surface $N$, then taking the orientation double-covering $\hat{N}$ of $N$, one obtains an embedding of the bipartite double-cover $\hat{G}$ in $\hat{N}$. However, the converse statement is false; if $\hat{G}$ embeds in $\hat{N}$, then it does not necessarily follow that $G$ embeds in $N$. Indeed, the graph $G$ shown on the left of Fig. 4 has the property that its bipartite double-cover $\hat{G}$ is planar ( $\hat{G}$ is shown on the right of Fig. 4; here the monodromy map is $\sigma(z)=-z$ ). Hence $\hat{G}$ embeds in the sphere $\mathbb{S}^{2}$. However $G$ does not admit a parity embedding in the real projective plane $\mathbb{R P}^{2}$. Indeed, if it did, the two triangles would both be represented by one-sided curves and hence both nontrivial in homology, and thus, as $H_{1}\left(\mathbb{R} P^{2}\right)=\mathbb{Z}_{2}$, they would be homologous curves. But in this case, they would necessarily have nontrivial intersection, which is impossible as they as disjoint.

In general, the problem is that given an embedding of $\hat{G}$ in a surface $\hat{N}$, it is not always possible to extend the action of the monodromy element $\sigma$ from $\hat{G}$ to a fixed point free involution of $\hat{N}$. Nevertheless, there is a partial result. Let $G$ be a connected 


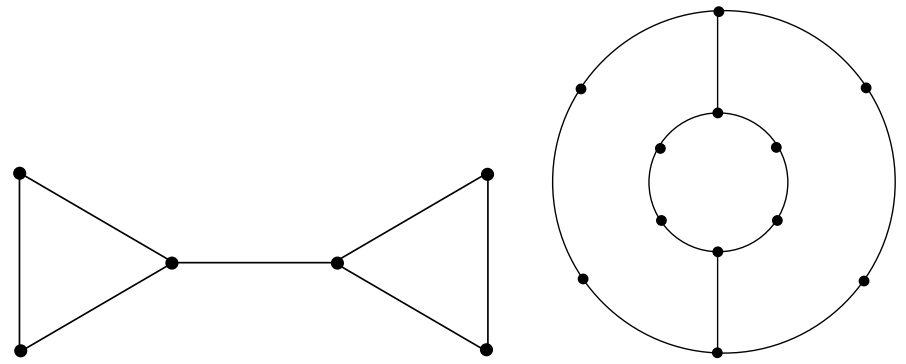

Fig. 4 The graph $G$ (on the left) and its bipartite double-cover $\hat{G}$

non-bipartite graph, let $\hat{G}$ denote its bipartite double-cover and let $\sigma: \hat{G} \rightarrow \hat{G}$ be the corresponding monodromy involution. Notice that if $R_{v}$ is rotation system at some vertex $v \in \hat{G}$, then the map $\sigma$ defines a rotation system $\sigma\left(R_{v}\right)$ at $\sigma(v)$; we call $\sigma\left(R_{v}\right)$ the image of $R_{v}$ under $\sigma$. We have:

Theorem 5 A finite graph $G$ admits a cellular parity embedding in a closed surface $N$ if and only if there is a cellular embedding of the bipartite double-cover $\hat{G}$ in the orientation cover $\hat{N}$ of $N$ with the following property: for each vertex $v$ of $\hat{G}$, the rotation system at $\sigma(v)$ is opposite to the image under $\sigma$ of the rotation system at $v$.

Proof One direction is obvious. Suppose therefore that there is a cellular embedding $\hat{\mathcal{E}}: \hat{G} \rightarrow \hat{N}$ with the property stated in the theorem. Notice that since the embedding is cellular, we can extend the involution $\sigma$ of $\hat{G}$ to an orientation reversing involutive homeomorphism $\hat{\sigma}$ of $\hat{N}$ by coning $\sigma$ on each face. Our task is to show that $\hat{\sigma}$ is fixed point free, since then $G=G^{\prime} / \sigma$ embeds in the closed surface $N=\hat{N} / \sigma$, and it is easy to see that this is a parity embedding and that $\hat{N}$ is the orientation cover of $N$.

Since $\hat{\sigma}$ maps faces to faces, and since $\sigma$ has no fixed point on $\hat{G}$, it suffices to show that $\hat{\sigma}$ preserves no face of $\hat{N}$. Arguing by contradiction, suppose that a face $F$ is preserved by $\hat{\sigma}$. Since $F$ is homeomorphic to a disc, and since $\hat{\sigma}$ is orientation reversing, $\hat{\sigma}$ induces an orientation reversing homeomorphism $\left.\hat{\sigma}\right|_{\partial F}$ on the boundary $\partial F \cong \mathbb{S}^{1}$ of $F$. Thus $\left.\hat{\sigma}\right|_{\partial F}$ has a fixed point (in fact, 2 fixed points), contradicting the fact that $\sigma$ has no fixed point on $\hat{G}$.

Remark 3 If $G$ can be drawn as a generalized thrackle on a closed orientable surface $M$, then by Theorem $2, G$ admits a parity embedding in a non-orientable closed surface $N$ with Euler characteristic $\chi(N)=\chi(M)-1$. We can then apply Theorem 5; notice that as the resulting surface $\hat{N}$ is a double cover of $N$, we have $\chi(\hat{N})=2 \chi(N)=2 \chi(M)-2$; see, for example, [4, Proposition IV.13.5].

Acknowledgements We thank Thomas Zaslavsky for his helpful comments and encouraging advice. We are also grateful to the paper's referees for their suggestions for improving the clarity of the presentation.

\section{References}

1. Archdeacon, D.: A Kuratowski theorem for the projective plane. J. Graph Theory 5, 243-246 (1981) 
2. Biggs, N.: Algebraic Graph Theory. Cambridge University Press, Cambridge (1974)

3. Berger, M., Gostiaux, B.: Differential Geometry: Manifolds, Curves, and Surfaces. Graduate Texts in Mathematics, vol. 115. Springer, New York (1988)

4. Bredon, G.E.: Topology and Geometry. Graduate Texts in Mathematics, vol. 139. Springer, New York (1997)

5. Cairns, G., Nikolayevsky, Y.: Bounds for generalized thrackles. Discrete Comput. Geom. 23, 191-206 (2000)

6. Cairns, G., McIntyre, M., Nikolayevsky, Y.: The thrackle conjecture for $K_{5}$ and $K_{3,3}$. In: Pach, J. (ed.) Towards a Theory of Geometric Graphs. Contemporary Mathematics. Am. Math. Soc., Providence (2004)

7. Diestel, R.: Graph Theory. Graduate Texts in Mathematics, vol. 173. Springer, Berlin (2005)

8. Dieudonné, J.: A History of Algebraic and Differential Topology. 1900-1960. Birkhäuser, Boston (1989)

9. Dubrovin, B.A., Fomenko, A.T., Novikov, S.P.: Modern Geometry, Part III. Springer, Berlin (1990)

10. Edelen, D.G.B.: Applied Exterior Calculus. Dover, New York (2005)

11. Giblin, P.J.: Graphs, Surfaces and Homology. Chapman \& Hall, Boca Raton (1981)

12. Gross, J.L., Tucker, T.W.: Topological Graph Theory. Dover, New York (2001)

13. Grove, L.C.: Classical Groups and Geometric Algebra. Graduate Studies in Mathematics, vol. 39. Am. Math. Soc., Providence (2002)

14. Lins, S.: Combinatorics of orientation reversing polygons. Aequ. Math. 29, 123-131 (1985)

15. Lovász, L., Pach, J., Szegedy, M.: On Conway's thrackle conjecture. Discrete Comput. Geom. 18, 368-376 (1997)

16. Mohar, B., Thomassen, C.: Graphs on Surfaces. Johns Hopkins Press, Baltimore (2001)

17. Perlstein, A., Pinchasi, R.: Generalized thrackles and geometric graphs in $\mathbb{R}^{3}$ with no pair of strongly avoiding edges. Preprint

18. Prasolov, V.V.: Elements of Combinatorial and Differential Topology. Graduate Studies in Mathematics, vol. 74. Am. Math. Soc., Providence (2006)

19. Woodall, D.R.: Thrackles and deadlock. In: Combinatorial Mathematics and Its Applications, pp. 335-347. Academic Press, San Diego (1971)

20. Woodall, D.R.: Unsolved problems. In: Combinatorics, Proc. Conf. Combinatorial Math., pp. 359363. Math. Inst., Oxford (1972)

21. Zaslavsky, T.: The projective-planar signed graphs. Discrete Math. 113, 223-247 (1993)

22. Zaslavsky, T.: The order upper bound on parity embedding of a graph. J. Comb. Theory Ser. B 68 , 149-160 (1996)

23. Zieschang, H., Vogt, E., Coldewey, H.-D.: Surfaces and Planar Discontinuous Groups. Lecture Notes in Mathematics, vol. 835. Springer, Berlin (1980) 